\title{
Breakage of an artificial crushable material under loading
}

\section{Francesca Casini, Giulia M. B. Viggiani \& Sarah M. Springman}

\section{Granular Matter}

ISSN 1434-5021

Volume 15

Number 5

Granular Matter (2013) 15:661-673 DOI 10.1007/s10035-013-0432-x

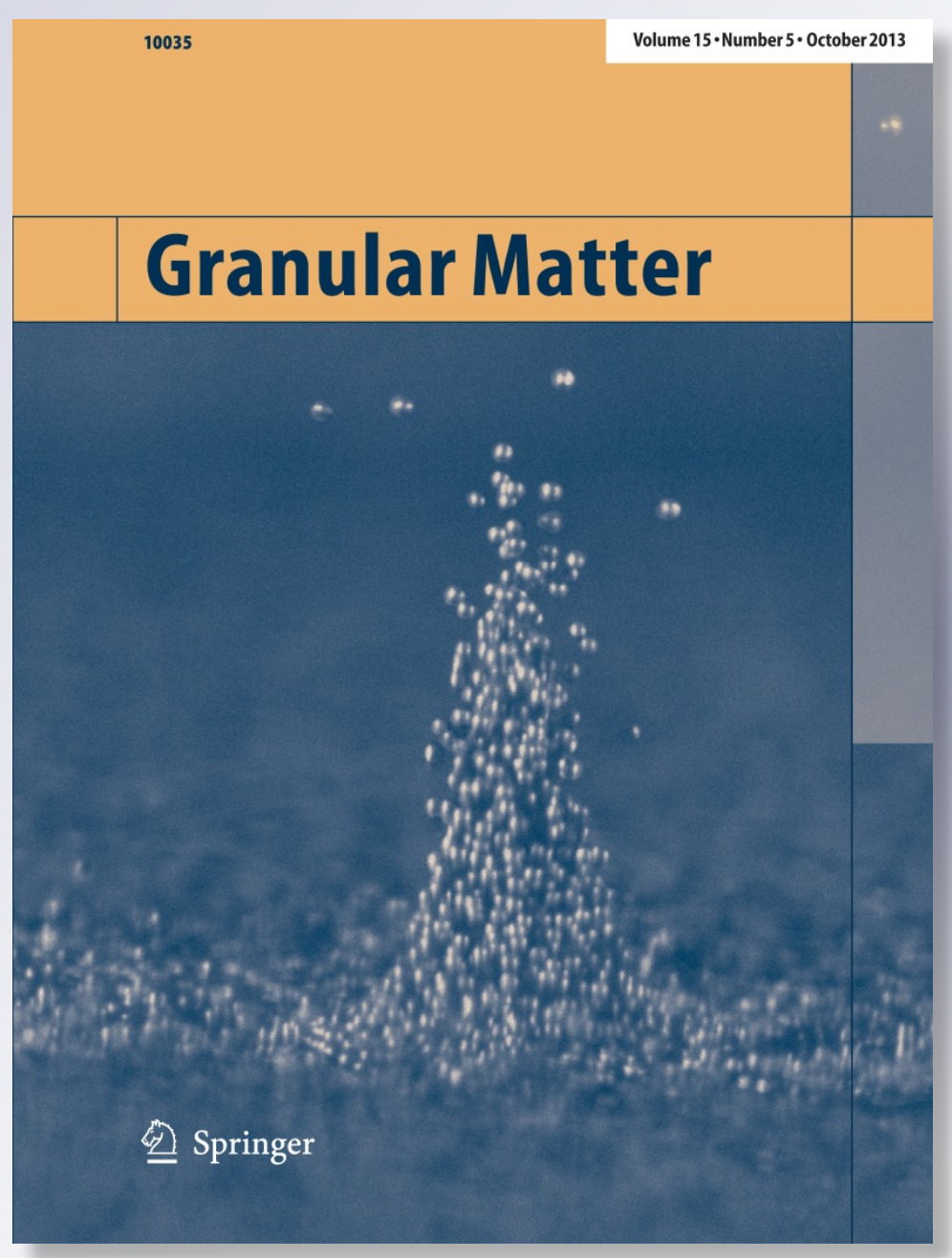

脰 Springer 
Your article is protected by copyright and all rights are held exclusively by SpringerVerlag Berlin Heidelberg. This e-offprint is for personal use only and shall not be selfarchived in electronic repositories. If you wish to self-archive your article, please use the accepted manuscript version for posting on your own website. You may further deposit the accepted manuscript version in any repository, provided it is only made publicly available 12 months after official publication or later and provided acknowledgement is given to the original source of publication and a link is inserted to the published article on Springer's website. The link must be accompanied by the following text: "The final publication is available at link.springer.com". 


\title{
Breakage of an artificial crushable material under loading
}

\author{
Francesca Casini - Giulia M. B. Viggiani • \\ Sarah M. Springman
}

Received: 16 October 2012 / Published online: 6 July 2013

(C) Springer-Verlag Berlin Heidelberg 2013

\begin{abstract}
The mechanical behaviour of granular materials depends on their grading. Crushing of particles under compression or shear modifies the grain size distribution, with a tendency for the percentage of fine material to increase. It follows that the frictional properties of the material and the critical states are modified as a consequence of the changes in grain size distribution and the available range of packing densities. This paper illustrates an extended experimental investigation of the evolution of the grading of an artificial granular material, consisting of crushed expanded clay pellets under different loading conditions. The changes of grading of the material after isotropic, one-dimensional and constant mean effective stress triaxial compression were described using a single parameter based on the ratio of the areas under the current and an ultimate cumulative particle size distribution, which were both assumed to be consistent with self similar grading with varying fractal dimension. Relative breakage was related to the total work input for unit of volume. For poorly graded samples, the observed maximum rate of breakage is practically independent of initial uniformity. Further
\end{abstract}

F. Casini $(\varangle)$

Departamento de Ingeniería del Terreno, Cartográfica y Geofísica, Universitat Politècnica de Catalunya, C. Jordi Girona 1-3, 08034 Barcelona, Spain

e-mail: francesca.casini@upc.edu

\section{G. M. B. Viggiani}

Dipartimento di Ingegneria Civile e Ingegneria Informatica, Università di Roma Tor Vergata, via del Politecnico 1, 00133 Rome, Italy

e-mail: viggiani@uniroma2.it

\section{S. M. Springman}

Institute for Geotechnical Engineering (IGT), ETH Zurich, Wolfgang-Pauli-Strasse 15, 8093 Zurich, Switzerland

e-mail: sarah.springman@igt.baug.ethz.ch experiments at higher confining stress are required to investigate the mechanics of breakage of better graded samples.

Keywords Granular material · Grain crushing · Grain size distribution $\cdot$ Fractals

\section{Introduction}

Loading of geotechnical structures may result in particle breakage of the constituent granular materials when the stress imposed on the particles exceeds their strength. From the perspective of applications in geotechnical engineering, this phenomenon is relevant in various engineering processes where crushing-related damage and internal instability are consequential, such as e.g.: detrimental clogging and formation damage near petroleum wells [12], permeability losses around perforations [10,31], impaired fluid conduction owing to particle crushing in locations adjacent to testing penetrometers and friction fatigue around piles [42], and crushing-induced instability transitions in highly stressed soil masses adjacent to or located within geotechnical structures, for instance filters for large dams [19]. [44] and [35] have discussed the effect of soil crushing on pile end bearing capacity while, more recently [22] examined the effect of particle crushing on the capacity of driven piles; Zheghal [45] studied the role of grain crushing in road construction; [29] reported that grain crushing within the failure zone is responsible for the rapid long run-out motion of landslides and sturzstroms, as observed in physical experiments in a geotechnical drum centrifuge by [16].

Particle breakage depends on a number of 'macro-scale' parameters, such as grading, voids ratio, state of effective stress, and effective stress path, as well as on the characteristics of the constituent particles, such as size, shape, strength, 
and mineral composition. The available experimental evidence, summarised, e.g.: by Lade et al. [18], seems to indicate that well-graded soils do not break down as easily as uniform soils. As the relative density increases, the amount of particle breakage decreases. Both of these observations are consistent with the fact that the average contact stress tends to decrease with more particles surrounding each particle. Several researchers have found that the amount of grain crushing under isotropic loading conditions is lower than under shearing $[17,23,28]$. As far as the characteristics of the constituent particles are concerned, it is well established that: (i) as the individual particle size increases, particle crushing increases, due to the fact that larger particles have a higher probability of containing defects or flaws; (ii) increasing the particle angularity increases particle breakage; (iii) increasing the mineral hardness decreases the amount of particle crushing.

Different measures have been suggested to quantify the amount of breakage undergone by a sample of granular material [13] introduced the relative breakage, $B_{\mathrm{r}}$, based on the shift of the current cumulative grain size distribution from the initial cumulative grain size distribution above a cut-off value of silt particle size $(0.074 \mathrm{~mm})$, with the implication that, in the fragmentation process, all particles would eventually become finer than this (arbitrary) cut-off value. This conflicts with the growing understanding that the grain size distribution of aggregates of any initial grading, under extremely large confining pressure and extensive shear strain, tends to a self-similar, fractal distribution $[25,37]$.

Several studies (e.g.: $[34,38,40]$ ) have also indicated that the predominant effect of particle crushing is to increase the proportion of fine material without significantly changing the size of the largest particles. To date, the mechanisms that cause the granular material to develop a fractal size distribution are not fully explained. According to [34], larger particles are more likely to be surrounded by smaller particles, which gives them higher coordination numbers and makes them more resistant to crushing, whereas smaller particles have smaller coordination numbers. In other words, the larger coordination number for larger particles outweighs the effect of the strength of individual particles reducing with increasing particle size. However, DEM simulations of direct shear tests on mixtures having a limited number of oversized particles carried out by [39] have shown that the smaller particles exert large compressive forces on a small section of the perimeter of the large particles [40] explain the fractal size distribution of the broken grains by the fact that compression of granular materials, simulated by DEM analyses, results in a network of force chains that are fractal in their intensity and distribution. It may be noted, however, that the numerical experiments were conducted on uniform granular materials with permanent grains.

Whatever the physical explanation for developing a fractal distribution of particle sizes under loading, this is potentially internally stable as it can be arranged in a fractal geometry, dispersing energy among the largest possible number of particle contacts and thus eliminating the occurrence of stress concentrations (e.g. $[15,16])$. Soils that exhibit fractal distributions are often considered internally stable and it has been suggested that a fractal distribution with a fractal dimension of around 2.5-2.6 [26,30,34] may be used in most practical cases. Fractal characteristics may not manifest over an infinite range of sizes, because particles smaller than a "comminution" limit do not fracture [24].

A number of recent detailed studies have considered the relationship between particle crushing and soil mechanical behaviour $([1,3-5,4,7,9,23,25,28,36])$. From the point of view of constitutive modelling, the question to be addressed is how microscopic degradation phenomena, such as grain crushing, affect the macroscopic properties of a granular aggregate, ideally deducing macroscopic constitutive equations from micromechanical consideration of some underlying microscopic process [25]. Quite recently, the idea of a fractal evolution of particle size has been adopted introducing a breakage parameter into energy dissipation assumptions of a crushable soil treated as a continuum [8], and through links between particle crushing and critical states in soils $[33,43]$.

Whatever the point of view adopted in constitutive modelling, the evolution of the grading of the material under different loading conditions must be examined experimentally to test the constitutive assumptions. This paper illustrates the results of an extensive experimental investigation of the evolution of the grading of an artificial granular material consisting of crushed expanded clay pellets under different loading conditions. The changes of grading of the material after isotropic, one-dimensional and triaxial compression were described using a single parameter based on the ratio of the areas under both the current and an ultimate cumulative particle size distribution, which were assumed to be consistent with self-similar grading with varying fractal dimension.

\section{Experimental work}

\subsection{Material tested}

The mechanical behaviour of many natural geotechnical materials, such as pyroclastic weak rocks, carbonate sands, calcarenites and residual soils, is affected by grain crushing. However, systematic experimental investigation of grain crushing for natural materials is often difficult due to the relatively high stress required to crush the grains and the variability and heterogeneity of natural deposits, which makes it difficult to obtain repeatable results. For these reasons, the experimental work was conducted on an artificial granular material consisting of crushed expanded clay pellets, 


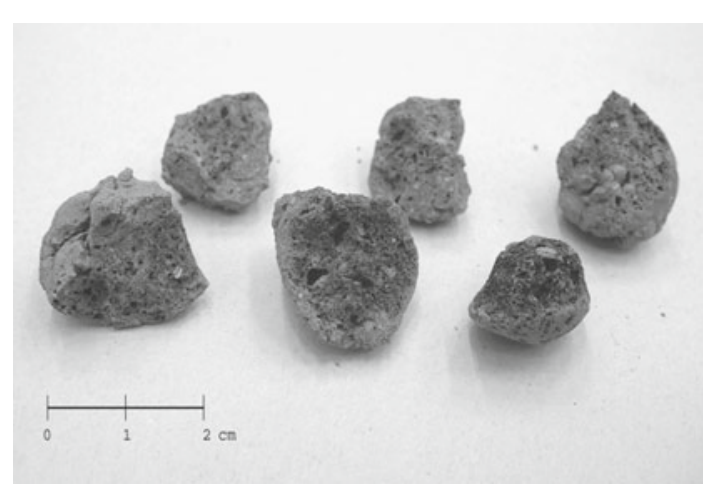

Fig. 1 Broken LECA pellets showing hard external shell and porous interior

commercially available under the acronym LECA (Light Expanded Clay Aggregate), whose grains break at relatively low stress. Lightweight expanded clay aggregates are used in road construction, tunnelling, structural backfill against foundations, retaining walls and bridge abutments, because of their low unit weight and good drainage properties. In many practical cases, the stress levels to which the material is subjected are comparable to those explored in this experimental investigation.

The material is obtained through an industrial process, in which the clay is homogenised, moistened, and broken up with grinding equipment and rolling mills. The main phase of the production cycle takes place in a long rotary kiln; the clay enters the kiln from one end and moves along it, gradually increasing its temperature. At the other end of the kiln, the temperature reaches approximately $1,200^{\circ} \mathrm{C}$, at which point the clay is in a molten state and the expansion process commences providing a cellular vitreous interior to each pellet. Rolling of the pellets within the kiln gives them a round shape and creates a hard outer shell (see Fig. 1). The expanded clay pellets are then screened into their various fractions and made commercially available both as intact (so-called "granular") or crushed, in different grain sizes.

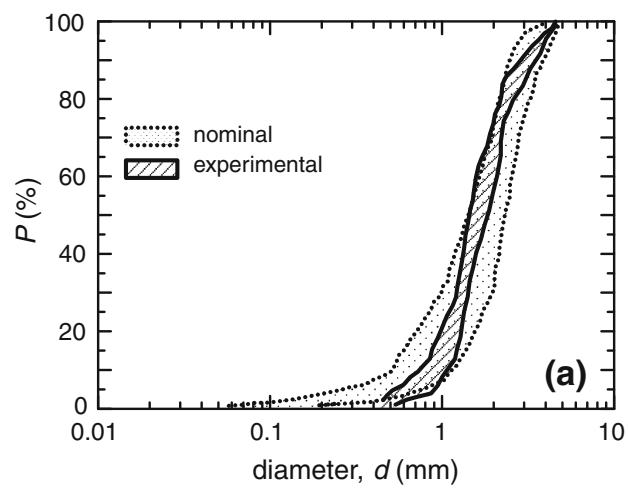

The cumulative grain size distributions by weight of samples of the material obtained from the producer in the finer grain sizes (0-2 and 2-4 mm for crushed material, and 04 and $4-6 \mathrm{~mm}$, for granular material) were determined by sieving and compared with the nominal ones [11]. Figure 2 shows that, while the grading curves obtained for the granular material were within the nominal range, those obtained for the crushed material manifested a significant increase of the percentage of material in the range of grain sizes between 0.1 and $0.2 \mathrm{~mm}$, and a reduction in the range between 0.2 and $0.5 \mathrm{~mm}$. This is likely to be due to further crushing occurred during carriage and handling, favoured by the angularity of the grains. Because of its high susceptibility to crushing, the experimental programme described in this work was carried out on crushed material in the $0-4 \mathrm{~mm}$ range of grain sizes.

In order to detect the structural and chemical components of the soil, a mineralogical investigation was carried out using X-ray diffractometry on the fraction with $d<2 \mu \mathrm{m}$. The main components were recognized as amorphous material (59.2\%), Quartz (20.6\%), Feldspar (10.2\%) and Spinel (10\%). No clay minerals were present, as these were destroyed at the high temperature reached in the kiln.

The material is characterised by a double order of porosity: "inter-granular", i.e., voids existing between particles, and "intra-granular", i.e., closed voids existing within individual particles (see Fig. 3). The significant intra-granular porosity is responsible for the low values of the apparent unit weight of the particles, $\gamma_{\text {as }}$ [2]. Figure 4 shows the values of $\gamma_{\text {as }}$ determined on different fractions of crushed material as a function of the fraction size; for $d<0.063 \mathrm{~mm}, \gamma_{\mathrm{as}}$ is equal to the unit weight of the constituent clay $\left(\gamma_{\mathrm{s}}=26.5 \mathrm{kN} / \mathrm{m}^{3}\right)$, while for $d$ larger than about $3.5 \mathrm{~mm}$, it tends to a constant value of about $9 \mathrm{kN} / \mathrm{m}^{3}$. For a particle size range of $0.063 \mathrm{~mm} \leq d<$ $3.5 \mathrm{~mm}$, which of interest in the present study, the experimental values of $\gamma_{\text {as }}$ were fitted with the following equation:

$\gamma_{\mathrm{as}}=a \cdot\left(d_{0} / d\right)^{b}$

with $a=12.64 \mathrm{kN} / \mathrm{m}^{3}, b=0.268$, and $d_{0}=1 \mathrm{~mm}$.

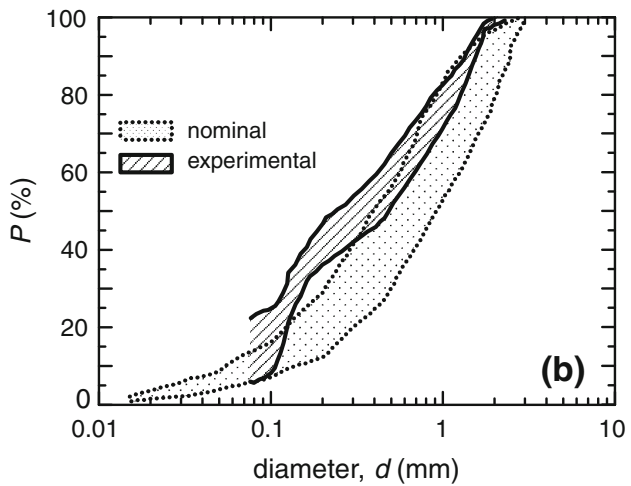

Fig. 2 Comparison of cumulative grain size distributions by weight of samples of $\mathbf{a}$ intact and $\mathbf{b}$ crushed material, as obtained from the producer, with the nominal distributions 
Fig. 3 Inter and intra-granular porosity (adapted from [14])

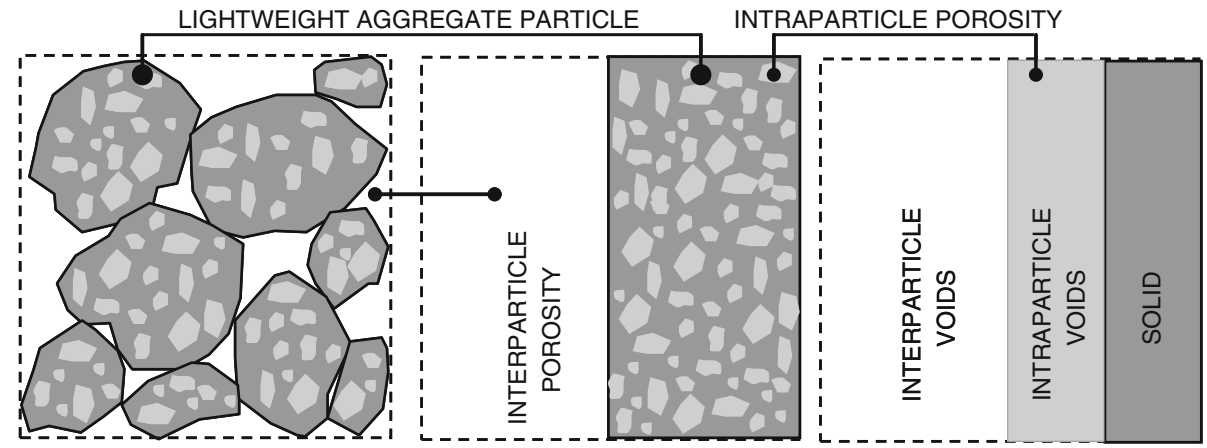

\subsection{Initial grading}

The material was reconstituted at different initial grain size distributions by weight, characterised by four values of the coefficient of uniformity $U\left(=d_{60} / d_{10}\right)=3.5,7,14$, and 28 and two values of mean grain size $d_{50}=0.5 \mathrm{~mm}$ and $1 \mathrm{~mm}$ (Fig. 5a).

For a material such as LECA, in which the apparent unit weight of particles depends on grain size, it is necessary to distinguish between grain size distribution by weight and grain size distribution by volume. The grain size distribution by weight is determined experimentally using a discrete series of sieves with dimension $\Delta_{i}(i=1,2, \ldots, n)$ to measure the weights of the particles that are finer than the sieve size $\Delta_{\mathrm{i}}$ and larger than the sieve size $\Delta_{i-1}, W_{i}=W\left(\Delta_{i-1}<\right.$ $d<\Delta_{i}$ ). On the one hand, the cumulative grain size distribution by weight is then computed as:

$P\left(d_{j}\right)=\frac{W\left(d<\Delta_{1}\right)+\sum_{2}^{j \leq n} W_{i}}{W_{\mathrm{T}}}$

in which $W\left(d<\Delta_{1}\right)$ is the material passing through all the sieves in the series and $W_{\mathrm{T}}=W\left(d<\Delta_{1}\right)+\sum_{2}^{n} W_{i}$ is the total weight of the sample. On the other hand, the volume of particles that are finer than the sieve size $\Delta_{i}$ and larger than the sieve size $\Delta_{i-1}$, can be computed as $V_{i}=W_{i} / \gamma_{\text {as } i}$, in which $\gamma_{\text {asi }}$ is the average apparent unit weight of the particles in the size range $\Delta_{i-1}<d<\Delta_{i}$. Using Eq. (1), this is:

$$
\begin{aligned}
\gamma_{\mathrm{as} i} & =\frac{1}{\Delta_{i}-\Delta_{i-1}} \int_{\Delta_{i-1}}^{\Delta_{i}} \gamma_{\mathrm{as}}(d) \mathrm{d} d \\
& =\frac{a\left(\Delta_{i}^{1-b}-\Delta_{i-1}^{1-b}\right)}{\left(\Delta_{i}-\Delta_{i-1}\right)(1-b)}
\end{aligned}
$$

and the cumulative grain size distribution by volume can be computed as:

$V\left(d_{j}\right)=\frac{W\left(d<\Delta_{1}\right) / \gamma_{\mathrm{as} 1}+\sum_{2}^{j \leq n} V_{i}}{V_{\mathrm{T}}}$

in which $\gamma_{\text {as1 }}$ is the average unit weight of the material with dimensions smaller than $\Delta_{1}$ and $V_{\mathrm{T}}=W(d<$

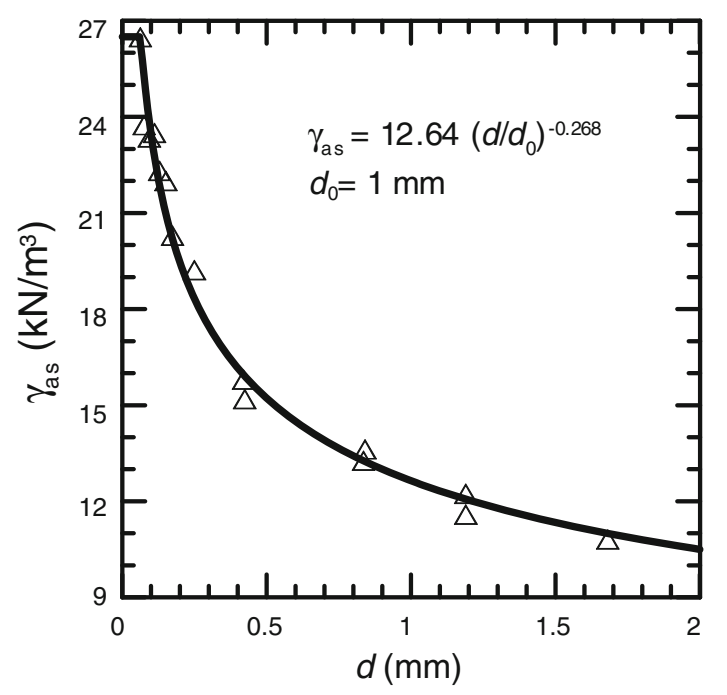

Fig. 4 Apparent unit weight of crushed LECA particles as a function of grain size

$\left.\Delta_{1}\right) / \gamma_{\text {as } 1}+\sum_{2}^{n} V_{i}$ is the total (apparent) volume of the solids in the sample. Figure $5 \mathrm{~b}$ shows the initial cumulative grain size distributions by volume of the tested material.

\subsection{Scanning electron microscopy (SEM)}

Figure 6a-f show examples of Scanning Electron Microscopy (SEM) micrographs of crushed LECA particles of different dimensions. SEM micrographs of portions of grains belonging to different fractions were manipulated using the image editing program GIMP [32]; the exposed intra-granular pores were coloured progressively in black and the contrast in the image was raised until all the pixels were either black (pores) or white (matrix), see Fig. 7. The processed images were then imported into Matlab and the number of white $\left(N_{\mathrm{W}}\right)$ and black $\left(N_{\mathrm{B}}\right)$ pixels counted with a simple algorithm; in this manner, it was possible to work out the exposed intragranular porosity of grains belonging to different fractions, $n_{\mathrm{ei}}=N_{\mathrm{B}} /\left(N_{\mathrm{B}}+N_{\mathrm{w}}\right)$. Figure 8 shows $n_{\mathrm{ei}}$ as a function of particle size, together with the bulk intra-granular porosity, obtained from the measurement of the apparent unit weight of particles of different sizes, $n_{\mathrm{bi}}\left(=1-\gamma_{\mathrm{as}} / \gamma_{\mathrm{s}}\right)$. The exposed 
Fig. 5 Initial cumulative grain size distribution of the tested material: $\mathbf{a}$ by weight and $\mathbf{b}$ by volume
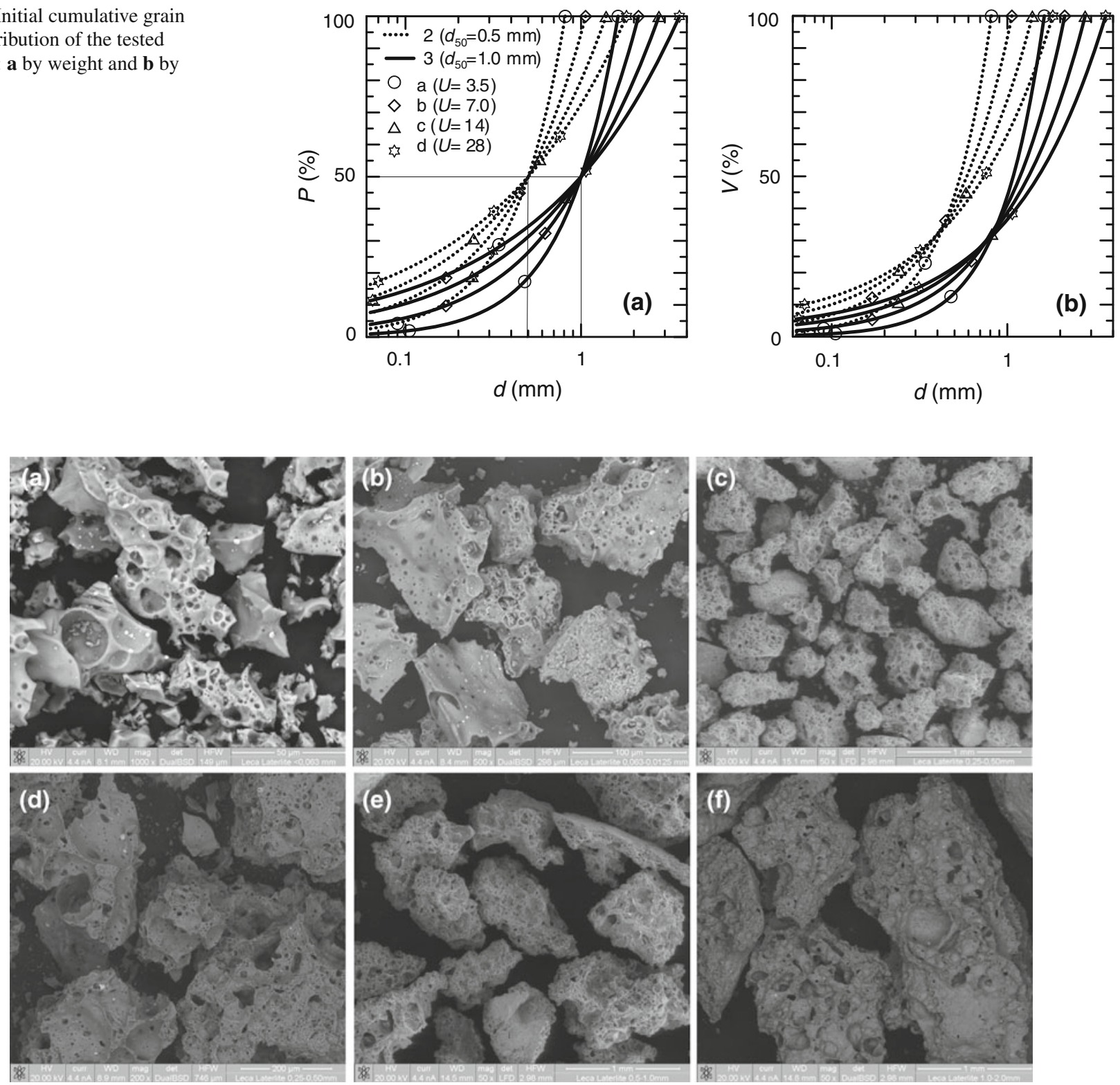

Fig. 6 SEM micrographs of crushed LECA particles of different size: a $0.063-0.125 \mathrm{~mm}, \mathbf{b} 0.125-0.25 \mathrm{~mm}, \mathbf{c} 0.25-0.5 \mathrm{~mm}$, d $0.5-1 \mathrm{~mm}$, e $1-2 \mathrm{~mm}$, and f $2-4 \mathrm{~mm}$

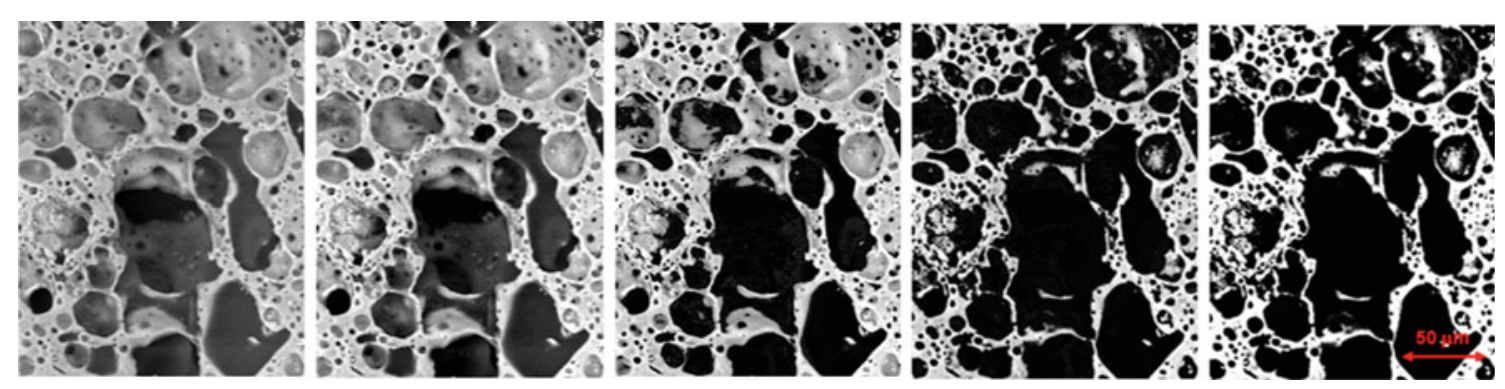

Fig. 7 An example demonstrating the steps followed in processing the SEM micrographs into a binary black-white distribution using the GIMP processing 


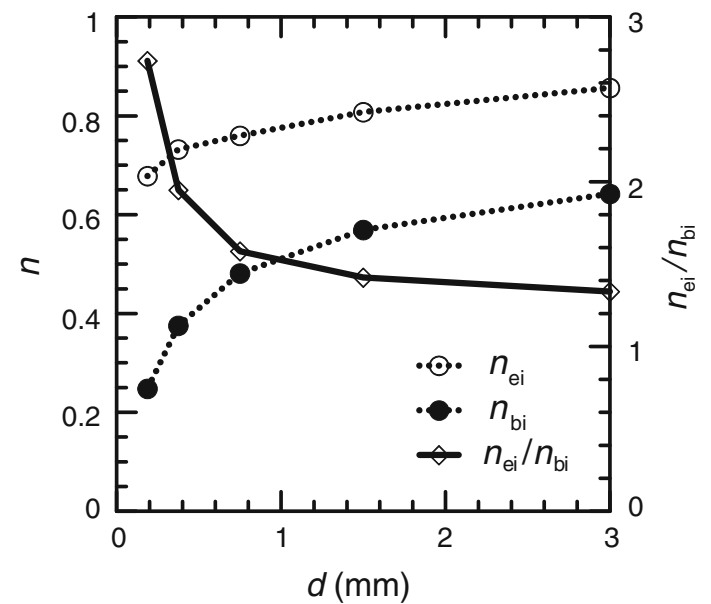

Fig. 8 Exposed and bulk intra-granular porosity of crushed LECA particles as a function of grain size

intra-granular porosity is always larger than the bulk intragranular porosity as the first is related to the ratio of the average void size to the particle size squared, while the second is related to the same ratio raised to a power 3 . Both $n_{\mathrm{ei}}$ and $n_{\mathrm{bi}}$ increase with increasing grain size, tending to constant values at particle sizes larger than about $3.5 \mathrm{~mm}$, where the apparent unit weight of the particles, $\gamma_{\mathrm{as}}$, becomes constant, with a final ratio $n_{\mathrm{ei}} / n_{\mathrm{bi}} \cong 1.3$.

\section{Experimental programme}

The experimental programme consisted of 64 tests including isotropic, one-dimensional and triaxial compression, carried out at increasing confining pressures, on reconstituted samples of each of the four initial grain size distributions [21, 37,41]. The experimental programme is detailed in Table 1; for one-dimensional compression, the values of maximum mean effective stress reported in Table 1 were computed as $p_{\text {max }}^{\prime}=\sigma_{\mathrm{v}, \text { max }}^{\prime}\left(1+2 K_{0}\right) / 3$ where $K_{0}=1-\sin \varphi^{\prime} \cong 0.55$.

\subsection{Dependence of physical properties on initial grading}

Prior to testing, the maximum and minimum intergranular voids ratio of each fraction, and of the eight grain size distributions adopted in the experimental work, were determined on dry material. The loosest packing was obtained by pouring the material into a mould using a funnel with drop height of zero; the densest packing was obtained by vibrating the material in the mould at very low energy to avoid breakage of the grains. The extent of the intergranular voids ratio, $e_{\max }-e_{\min }$, for crushed LECA at different values of $U$ and $d_{50}$ by volume, were compared with those reported by [27] for a wide range of granular materials, including natural sands, light-
Table 1 Experimental programme

\begin{tabular}{|c|c|c|c|c|c|c|c|}
\hline \multirow[t]{3}{*}{ Type } & \multicolumn{3}{|c|}{ Initial grading $^{\mathrm{a}}$} & \multicolumn{4}{|c|}{$p_{\max }^{\prime}(\mathrm{kPa})$} \\
\hline & & \multirow[t]{2}{*}{$\mathrm{U}(-)$} & \multirow[t]{2}{*}{$d_{50}(\mathrm{~mm})$} & 1 & 2 & 3 & 4 \\
\hline & & & & 175 & 350 & 700 & 1,400 \\
\hline ISO & $2 \mathrm{a}$ & 3.5 & 0.50 & $\bullet$ & $\bullet$ & $\bullet$ & \\
\hline ISO & $2 \mathrm{c}$ & 14 & 0.50 & $\bullet$ & $\bullet$ & & \\
\hline ISO & $2 d$ & 28 & 0.50 & $\bullet$ & $\bullet$ & & \\
\hline ISO & $3 a$ & 3.5 & 1.00 & $\bullet$ & $\bullet$ & & \\
\hline ISO & $3 c$ & 14 & 1.00 & $\bullet$ & $\bullet$ & $\bullet$ & \\
\hline ISO & $3 d$ & 28 & 1.00 & $\bullet$ & $\bullet$ & $\bullet$ & \\
\hline OED & $2 \mathrm{a}$ & 3.5 & 0.50 & $\bullet$ & $\bullet$ & $\bullet$ & $\bullet$ \\
\hline OED & $2 b$ & 7 & 0.50 & $\bullet$ & $\bullet$ & $\bullet$ & $\bullet$ \\
\hline OED & $2 \mathrm{c}$ & 14 & 0.50 & $\bullet$ & $\bullet$ & $\bullet$ & $\bullet$ \\
\hline OED & $2 d$ & 28 & 0.50 & $\bullet$ & $\bullet$ & $\bullet$ & $\bullet$ \\
\hline OED & $3 a$ & 3.5 & 1.00 & $\bullet$ & $\bullet$ & $\bullet$ & $\bullet$ \\
\hline OED & $3 b$ & 7 & 1.00 & $\bullet$ & $\bullet$ & $\bullet$ & $\bullet$ \\
\hline OED & $3 c$ & 14 & 1.00 & - & $\bullet$ & $\bullet$ & $\bullet$ \\
\hline OED & $3 d$ & 28 & 1.00 & $\bullet$ & $\bullet$ & • & - \\
\hline TXC & $2 \mathrm{a}$ & 3.5 & 0.50 & $\bullet$ & & $\bullet$ & \\
\hline TXC & $2 \mathrm{c}$ & 14 & 0.50 & $\bullet$ & $\bullet$ & $\bullet$ & \\
\hline TXC & $2 d$ & 28 & 0.50 & $\bullet$ & $\bullet$ & $\bullet$ & \\
\hline TXC & $3 a$ & 3.5 & 1.00 & $\bullet$ & $\bullet$ & $\bullet$ & \\
\hline TXC & $3 c$ & 14 & 1.00 & $\bullet$ & $\bullet$ & $\bullet$ & \\
\hline TXC & $3 d$ & 28 & 1.00 & $\bullet$ & $\bullet$ & $\bullet$ & \\
\hline
\end{tabular}

${ }^{\mathrm{a}}$ Grain size distribution by weight

weight aggregates (LWA) and glass beads. The experimental data indicate that $e_{\max }-e_{\min }$ decreases slightly with both $d_{50}$ and $U$ (see Fig. 9a, b); however, the values obtained for crushed LECA are much larger than those obtained for other granular materials. These very high values of the range of voids ratio at which it is possible to reconstitute the material are due to the very light weight, angularity and rough surface of the particles of crushed material, which favours a very "open" structure [20].

The constant volume friction angle of crushed LECA was determined by forming a cone of sand through pluviation in air and measuring the inclination of the slope formed; the resulting values for the eight grain size distributions adopted in the experimental work are of the order of $30^{\circ}-33^{\circ}$. The angle of repose $\left(\varphi_{\mathrm{cv}}^{\prime}\right)$ is almost constant with $d_{50}$ and $U$ (see Fig. 9c, d), and the experimental values are in good agreement with those measured on other granular materials with permanent grains [27].

\subsection{Evolution of grading with loading}

Figure 10 shows some general characterisation of the evolution of grading with loading, using data obtained from sam- 

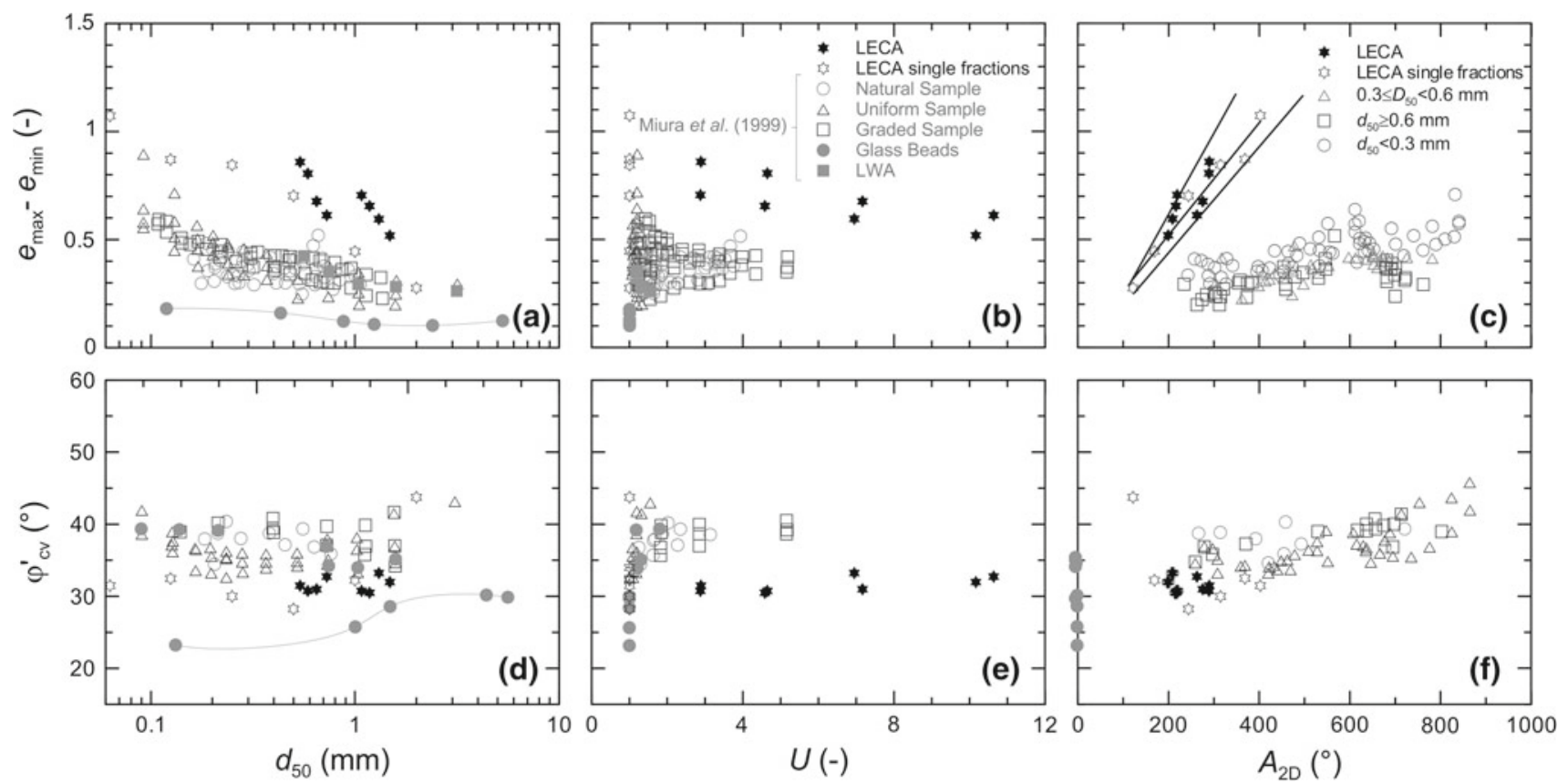

Fig. 9 Comparison of basic properties between permanent grain and crushable materials
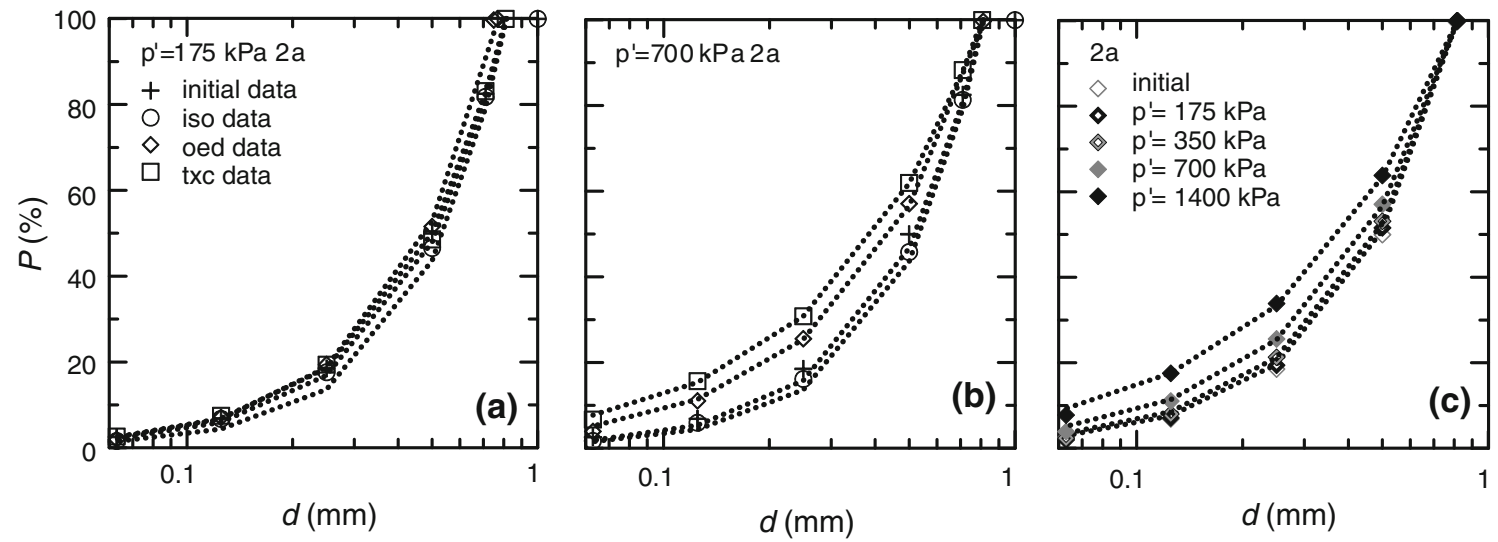

Fig. 10 Grain size distribution evolution for $d_{50 \mathrm{i}}=0.50 \mathrm{~mm}$ and $U=3.5$, for a variety of laboratory compression tests on LECA: a $p^{\prime}=175 \mathrm{kPa}$; b $p^{\prime}=750 \mathrm{kPa}$; 1 -D compression

ples with an initial grading with $d_{50 \mathrm{i}}=0.5 \mathrm{~mm}$ and $U_{i}=3.5$. Figure $10 \mathrm{a}, \mathrm{b}$ shows the grain size distribution by weight obtained after isotropic, one-dimensional and triaxial compression after reaching either measured or calculated final values of the mean effective stress equal to 175 and $700 \mathrm{kPa}$, respectively. In both cases, the cumulative grain size distribution at the end of the test is modified by loading, but the changes in the grain size distribution are hardly detectable at $p^{\prime}=175 \mathrm{kPa}$, and much more pronounced at $p^{\prime}=700 \mathrm{kPa}$. The data in Fig. 10b shows that the amount of grain crushing increases with the maximum obliquity $\eta\left(=q / p^{\prime}\right)$ reached in the test; isotropic compression, even at relatively large mean effective stress, causes only very limited changes to the cumulative grain size distribution. Figure 10c shows the grain size distribution obtained after one-dimensional compression at increasing values of the maximum mean effective stress reached in the test, from 175 to $1,400 \mathrm{kPa}$; in all cases the final grain size distribution is rotated upwards and translated leftwards, with an increase in the fine fraction at an almost constant value of the maximum particle size, $d_{\mathrm{M}}$. The maximum particle size $d_{\mathrm{M}}$ is likely to be different from $\Delta_{\mathrm{n}}$ (maximum dimension of the sieve series) and unknown, even if, of course, $d_{\mathrm{M}} \leq \Delta_{\mathrm{n}}$. Small changes of $d_{\mathrm{M}}$ with load and stress path are difficult to detect in the laboratory because the spacing of two sieves of successive size around $d_{\mathrm{M}}$ is finite, and not fine enough. 
Fig. 11 Evolution with mean effective stress of: a $d_{50}$; b uniformity $U$

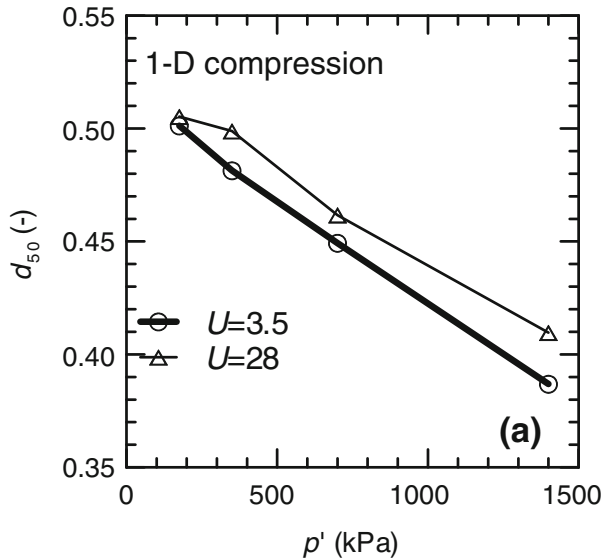

As a first approximation, the amount of grain crushing may be quantified using the variation of the mean grain size $d_{50}$ or the coefficient of uniformity $U$ after loading. Figure $11 \mathrm{a}, \mathrm{b}$ shows the evolution of $d_{50}$ and $U$ for the two extreme values of the initial uniformity tested $\left(U_{i}=3.5\right.$ and 28) as a function of maximum mean effective stress $p^{\prime}$ after one-dimensional compression. The reduction of $d_{50}$ and the increase of $U$ with increasing mean effective stress are slightly more pronounced for smaller initial uniformity coefficient and larger mean diameter.

\section{Analysis of results}

For a fractal grain size distribution, the number of particles that have dimension $\Delta$ larger than $d$ is $N(\Delta>d)=C d^{-\alpha}$ in which $C$ is a constant and $\alpha$ is the fractal dimension [37], while the number of particles in a fraction is [26]:

$d N(\Delta)=\alpha C \Delta^{-\alpha-1} d \Delta$

The (continuous) cumulative grain size distribution by volume is equal to:

$V(d)=\frac{V(d<\Delta)}{V_{\mathrm{T}}}=\frac{\int_{0}^{d} s \Delta^{3} d N(\Delta)}{\int_{0}^{d_{\mathrm{M}}} s \Delta^{3} d N(\Delta)}$

in which $s$ is a shape factor and $d_{\mathrm{M}}$ is the largest particle size in the sample. Substituting Eq. (5) into Eq. (6), the following expression for the cumulative grain size distribution by volume of a fractal grain size distribution is obtained:

$V(d)=\left(\frac{d}{d_{\mathrm{M}}}\right)^{3-\alpha}=\left(\frac{d}{d_{\mathrm{M}}}\right)^{\beta}$

If the apparent unit weight of particles depends on grain size as per Eq. (1), it is easy to show that the corresponding cumulative grain size distribution byweight is:

$$
\begin{aligned}
P(d) & =\frac{W(\Delta<d)}{W_{\mathrm{T}}}=\frac{\int_{0}^{d} s \gamma_{\mathrm{as}}(\Delta) \Delta^{3} d N(\Delta)}{\int_{0}^{d_{\mathrm{M}}} s \gamma_{\mathrm{as}}(\Delta) \Delta^{3} d N(\Delta)} \\
& =\left(\frac{d}{d_{\mathrm{M}}}\right)^{3-\alpha-\mathrm{b}}=\left(\frac{d}{d_{\mathrm{M}}}\right)^{\beta_{\mathrm{w}}}
\end{aligned}
$$

in which $\beta_{\mathrm{w}}=\beta-b$. For a material in which the apparent unit weight does not depend on grain size $(b=0)$, the cumulative grain size distribution by weight and by volume are the same $\left(\beta_{\mathrm{w}}=\beta\right)$.

In the interpretation of data, it is assumed that the ultimate grain size cumulative distribution by volume is fractal:

$V_{u}(d)=\left(\frac{d}{d_{M \mathrm{u}}}\right)^{\beta_{\mathrm{u}}}$

In the derivations above, it is also implicitly assumed that the minimum grain size dimension is $d_{\mathrm{m}}=0$, i.e. that there is no comminution limit to the grain size.

It is further assumed that the initial and current (evolving) cumulative grain size distributions by volume may be written in a similar form to that of the ultimate grain size distribution:

$V_{\mathrm{i}}(d)=\left(\frac{d}{d_{M_{\mathrm{i}}}}\right)^{\beta_{i}} \quad V(d)=\left(\frac{d}{d_{M}}\right)^{\beta}$

where $d_{\mathrm{Mi}}$ and $\beta_{i}$ represent the values of $\beta$ and $d_{\mathrm{M}}$ respectively for the $i$-th range of sieve sizes. Although the initial cumulative grain size distribution may not be self-similar, in most practical cases the initial cumulative grain size distributions would be reasonably fitted by an equation similar to (14) and the loading required to reduce the initial distribution to a self-similar distribution would be small. For instance, even the very small variations of grading obtained under isotropic compression, significantly improve the best fit of the grain size distribution using a self-similar equation; this is also confirmed by the experimental observations of the evolution of grading with loading of granular materials with very low values of the initial coefficient of uniformity [9]. 
Both parameters defining Eq. (10), namely $\beta$ and $d_{\mathrm{M}}$, were obtained by regression of the experimental data; it turned out that, in the range of mean effective stress examined, $d_{\mathrm{M}}$ is almost constant, with a maximum observed variation of only about $9 \%$, while $\beta$ reduces significantly due to grain crushing, with a maximum of about $33 \%$.

Figure 12 shows the evolution of parameters $\beta$ and $d_{\mathrm{M}}$ with the maximum obliquity $\eta_{\max }$ attained in the tests at the minimum and maximum value of mean effective stress used for $d_{50 \mathrm{i}}=0.5 \mathrm{~mm}, p^{\prime}=175$ and $700 \mathrm{kPa}$, respectively. Particle breakage manifests mainly as a reduction of $\beta$ and, to a lesser extent, of $d_{\mathrm{M}}$ with increasing obliquity. The reduction of $\beta$ with $\eta_{\max }$ is very evident at $p^{\prime}=700 \mathrm{kPa}$ and very small at $p^{\prime}=175 \mathrm{kPa}$. Poorly graded samples crush more than more uniform samples, with a more pronounced decrease of $\beta$ as $U_{i}$ increases. At the same time, the decrease of $d_{\mathrm{M}}$ with increasing $\eta_{\max }$ is more pronounced for larger values of $U_{\mathrm{i}}$; this likely to be due to the fact that, at the same value of $d_{50 \mathrm{i}}$, well graded samples contain particles of larger size with a higher probability of containing defect or flaws (e.g. [6]).

Figure 13 shows the observed variation of $\beta$ and $d_{\mathrm{M}}$ as a function of the maximum mean effective stress $p_{\max }^{\prime}$ attained during one-dimensional compression for the eight tested cumulative initial grain size distribution (see Fig. 5; Table 1). The data show clearly that $\beta$ decreases with increasing $p_{\max }^{\prime}$, for all values of initial uniformity and both values of initial mean grain size; the reduction of $\beta$ with $p_{\max }^{\prime}$ is more pronounced for initially poorly graded samples. The reduction of $d_{\mathrm{M}}$ with $p_{\max }^{\prime}$ can be detected only for more well graded samples $\left(U_{i}=28\right)$ both for $d_{50 \mathrm{i}}=0.5$ and $1 \mathrm{~mm}$.

Based on the data discussed above, it is assumed in the following sections that the maximum grain dimension is constant $\left(d_{\mathrm{Mi}}=d_{\mathrm{M}}=d_{\mathrm{Mu}}\right)$, while the exponent $\beta$ evolves with loading. The minimum observed value of $\beta$ is taken to be the exponent of the ultimate cumulative grain size distribution $\left(\beta_{u}=0.64\right)$. The description of breakage through only one scalar parameter $\beta$, conceptually similar to Einav's [8] assumption of fractional independency, is equivalent to the use of a single internal variable in elastic-plastic models for granular media with crushable grains.

\section{Dependence of breakage on work input}

Einav [8] suggested the original definition of the relative breakage by [13] should be adjusted to weigh the relative proximity of the current grain size cumulative distribution from the initial cumulative distribution and an ultimate cumulative distribution from zero to one. Following this approach, relative breakage can be obtained by computing the areas below the current, initial, and ultimate cumulative grain size distributions by integration over the logarithm of $d$ (after [8]):

$$
\begin{aligned}
& A=\int_{0}^{d_{M}} V(d) \mathrm{d}(\log d)=\frac{1}{\ln 10} \int_{0}^{d_{M}} V(d) d^{-1} \mathrm{~d} d=\frac{0.434}{\beta} \\
& A_{\mathrm{i}}=\frac{0.434}{\beta_{\mathrm{i}}} A_{\mathrm{u}}=\frac{0.434}{\beta_{\mathrm{u}}}
\end{aligned}
$$

The breakage potential, $B_{\mathrm{p}}$, and total breakage, $B_{t}$, can then be obtained by subtracting the area below the initial grain size distribution from the area below the ultimate grain size distribution or below the current grain size distribution (see Fig. 14):

$B_{p}=A_{u}-A_{\mathrm{i}}=0.434 \cdot\left(\frac{1}{\beta_{u}}-\frac{1}{\beta_{\mathrm{i}}}\right)$

and:

$B_{t}=A-A_{\mathrm{i}}=0.434 \cdot\left(\frac{1}{\beta}-\frac{1}{\beta_{\mathrm{i}}}\right)$

and, finally, the relative breakage is obtained as:

$B_{r}=\frac{B_{t}}{B_{p}}=\frac{A-A_{\mathrm{i}}}{A_{u}-A_{\mathrm{i}}}=\left(\frac{1}{\beta}-\frac{1}{\beta_{\mathrm{i}}}\right) /\left(\frac{1}{\beta_{u}}-\frac{1}{\beta_{\mathrm{i}}}\right)$

Given the ultimate grain size cumulative distribution, the relative breakage is limited by $0 \leq B_{\mathrm{r}} \leq 1$, where $B_{\mathrm{r}}=0$ denotes unbroken material and $B_{\mathrm{r}}=1$ represents complete breakage.

Following the approach by [43], a single reference initial cumulative distribution was adopted in this work as a vertical line through $d_{\mathrm{M}}$. This corresponds to assuming that the reference initial cumulative grain size distribution is the Heaviside function around $d_{\mathrm{M}}\left(\beta_{0} \rightarrow \infty ; A_{0} \rightarrow 0\right)$. In this way, $B_{\mathrm{r}}$ still spans from zero to unity as $\beta$ spans from $\beta_{0}(\rightarrow \infty)$ to $\beta_{u}$, although the initial grading of the sample corresponds to a non-zero initial value of $B_{\mathrm{r}}$. In most practical cases, even a very uniformly graded sample would have a high but finite value of $\beta_{i}(\cong 50-60)$, and therefore a finite, non-zero value of relative breakage, $B_{\mathrm{ri}}$, using the universal initial grading definition. The relative breakage is therefore $B_{\mathrm{r}}=A / A_{\mathrm{u}}=\beta_{\mathrm{u}} / \beta$.

Figure 15 shows the relative breakage obtained at the end of the different tests as a function of the work input for a unit of volume, $L=p^{\prime} \Delta \varepsilon_{\mathrm{v}}+q \Delta \varepsilon_{\mathrm{q}}$, in which $\Delta \varepsilon_{\mathrm{v}}$ and $\Delta \varepsilon_{\mathrm{q}}$ are the increments of volumetric and deviatoric strain, respectively. The experimental data in Fig. 15 are fitted with curves of equation:

$$
\left(1-B_{\mathrm{r}}\right) /\left(1-B_{\mathrm{ri}}\right)=\left[1+m\left(L / L^{*}\right)^{1 /(1-m)}\right]^{-m}
$$

where $L^{*}$ and $m$ are model parameters. It was found that parameter $L^{*}$ is a material constant, in this case with a value $L^{*}=100 \mathrm{kNm} / \mathrm{m}^{3}$ for LECA, while $m$ increases with the initial uniformity. 
Fig. 12 Evolution of the parameters a $\beta$; $\mathbf{b} d_{\mathrm{M}}$ with $\eta_{\max }$ for $p_{\max }^{\prime}=175 \mathrm{kPa}$ (open symbols) and $700 \mathrm{kPa}$ (shaded symbols)
Fig. 13 Evolution of the parameters: $\mathbf{a} \beta$; b $d_{\mathrm{M}}$ with $p_{\max }^{\prime}$ in 1-D compression for $d_{50}=0.5 \mathrm{~mm}$ (open symbols) and $d_{50}=1.0 \mathrm{~mm}$ (shaded symbols)
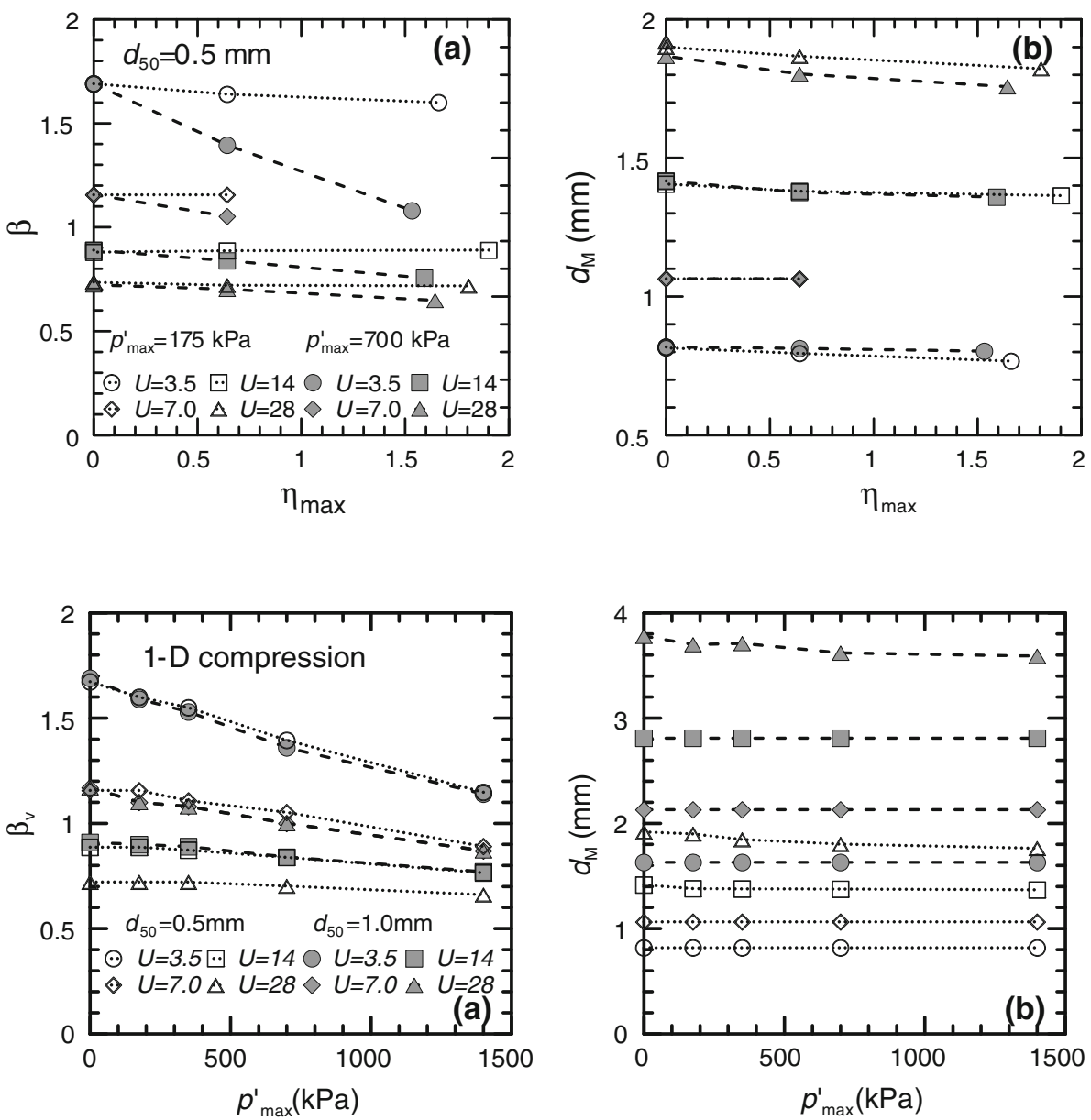

The rate of breakage, $\mathrm{d} B_{\mathrm{r}} / \mathrm{dL}$ :

$$
\begin{aligned}
& \frac{\mathrm{d} B_{\mathrm{r}}}{\mathrm{d} L}=\left(1-B_{\mathrm{ri}}\right) \\
& \cdot \frac{m^{2}\left(L / L^{*}\right)^{1 /(1-m)}\left[\left(m\left(L / L^{*}\right)^{1 /(1-m)}+1\right)\right]^{-m-1}}{L(1-m)}
\end{aligned}
$$

is represented in Fig. 16. $L^{*}$ represents the work input at which the rate of breakage, $\mathrm{dBr} / \mathrm{dL}$, is maximum and, when substituted into Eq. 16, is equal to:

$$
\left(\frac{\mathrm{d} B_{\mathrm{r}}}{\mathrm{d} L}\right)_{\max }=\frac{1-B_{\mathrm{ri}}}{L^{*}} \cdot \frac{m^{2}(m+1)^{-m-1}}{1-m}
$$

The observed maximum rate of breakage is practically independent of initial uniformity, up to $U_{i} \cong 14$, while it is significantly smaller for more well graded samples ( $\left.U_{i}=28\right)$. This is consistent with the observation that there is a more pronounced decrease of $\beta$ with loading for poorly graded samples (see Figs. 12, 13). As observed above, particle breakage manifests also with a small reduction of $d_{\mathrm{M}}$ with loading, which is neglected in the model; the reduction of $d_{\mathrm{M}}$, however, can be detected only for more well graded samples, whose initial uniformity is closer to the uni-

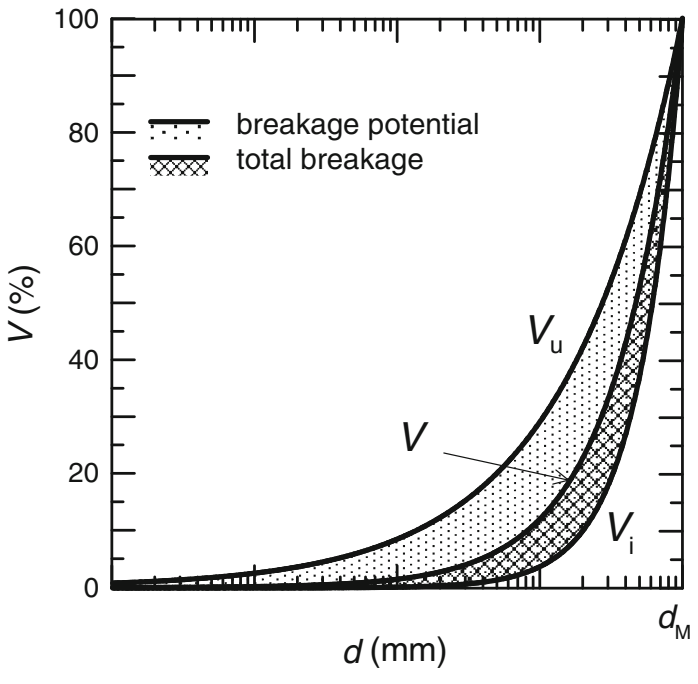

Fig. 14 Definition of breakage potential and total breakage

formity of the ultimate grading. These samples have an initial grain size distribution with a fractal dimension by weight of 2.56, within the suggested range of values by [34], or, in other words, a value of exponent $\beta$, which is closer to the 


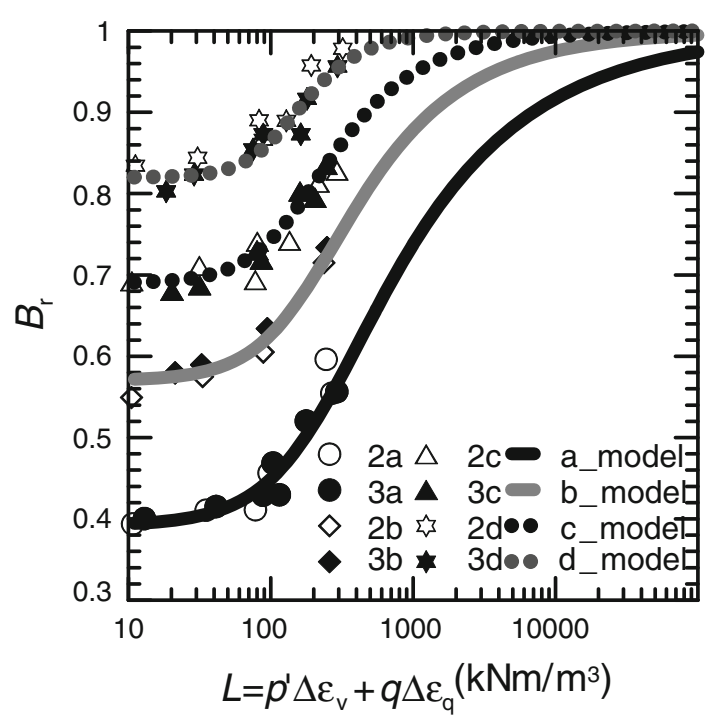

Fig. 15 Relative breakage as a function of work input per unit volume

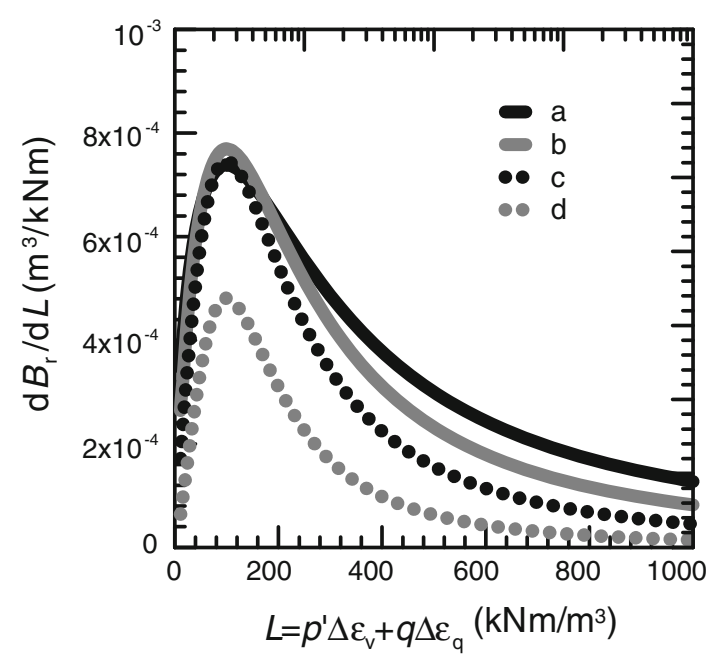

Fig. 16 Rate of breakage as a function of work input per unit volume

ultimate value. For a sample that has such an initial grain size distribution, the only way in which the grading may evolve is by a concurrent reduction of the maximum particle size in the sample, but this may require higher stress levels. Further experiments at higher confining stress are required to investigate the mechanics of breakage of better graded samples.

\section{Conclusions}

This paper illustrates the results of an extended experimental investigation of the evolution of grain size distribution due to loading along different stress paths. The final goal of the research is to gain a deeper understanding of the mechanical behaviour of soils with crushable grains, but also of weak rocks, or cemented aggregates whose bonds suffer progressive degradation due to applied loads, in order to develop suitable constitutive equations that incorporate the (evolving) grain size distribution.

The experimental work was carried out on an artificial material composed of crushed expanded clay pellets, that break at relatively low stress, reconstituted to obtain repeatable grain size distributions with different values of $U(=$ $\left.d_{60} / d_{10}\right)=3.5,7,14$, and 28 and two values of mean grain size $d_{50}=0.5$ and $1 \mathrm{~mm}$. The grain size distributions were measured after isotropic, one-dimensional and triaxial compression at increasing stress levels and compared with the initial grading of the samples. A total of 64 tests were carried out at maximum mean effective stress ranging between 175 and $1,400 \mathrm{kPa}$.

Simple measures of grain crushing, such as the reduction of $d_{50}$ or the increase of the uniformity coefficient $U$, demonstrate that crushing depends both on the stress ratio $\eta$ and on the value of mean effective stress $p$ ' reached in the test.

In the analysis of the results, the ultimate, current, and initial cumulative grain size distribution by volume were all taken to be self-similar, with a constant value of the maximum grain dimension, $d_{\mathrm{M}}$, and an evolving exponent, $\beta$. The description of breakage through only one scalar parameter $\beta$, is conceptually similar to Einav's [8] assumption of fractional independency.

The relative breakage, $B_{\mathrm{r}}$, describing the evolution of grain size distribution with loading, has been defined following the approach by [43], i.e., a single reference initial cumulative distribution as a vertical line through $d_{\mathrm{M}}$ was adopted, with the implication that the initial grading of the samples corresponds to a non-zero initial value of $B_{\mathrm{r}}$.

Granular materials undergo several dissipative mechanisms in the loading process, which include energy dissipation from the breakage of the particles (here described via $B_{\mathrm{r}}$ and hence by exponent $\beta$ ), but also from granular rearrangement with relative frictional sliding and rotation. As a first approximation, the values of relative breakage have been related to the total work input for unit of volume using an equation defined by two parameters, namely $L^{*}$ and $m . L^{*}$ represents the work input at which the rate of breakage, $\mathrm{d} B_{\mathrm{r}} / \mathrm{d} L$, is maximum and equal to a value that depends on $m$ and on the initial uniformity. $L^{*}$ is a material constant while $m$ increases with the initial uniformity.

For poorly graded samples (up to $U_{i}=14$ ), the observed maximum rate of breakage is practically independent of initial uniformity. For more well graded samples $\left(U_{i}=28\right)$, the maximum rate of breakage is smaller; in this case, however, particle breakage is manifest with a concurrent reduction of $\beta$ and $d_{\mathrm{M}}$ with loading, which is neglected in the model. Further experiments at higher confining stress are required to investigate the mechanics of breakage of more well graded samples. 
Acknowledgments Part of this research was carried out while the first Author was a post-doc at ETH Zurich. The authors wish to acknowledge Flavio Wanninger, Pascal Zwicker, Jacqueline Leu, Barbara Low, and Annika Zimmermann, who carried out some of the experimental work in partial fulfillment of the requirements for their Semester projects during their Masters studies at ETH Zürich, and the technical support of Ralf Herzog and Gabriele Peschke.

\section{References}

1. Arslan, H., Baykal, G., Sture, S.: Analysis of the influence of crushing on the behaviour of granular materials under shear. Granul. Matter 11, 87-96 (2009)

2. Casini, F., Viggiani, G.M.B.: Experimental investigation of the evolution of grading of an artificial material with crushable grains under different loading conditions. In: Proceedings of the International Symposium on Deformation Characteristics of Geomaterials IS-Seoul, pp. 957-964 (2011)

3. Cecconi, M., Viggiani, G.: Physical and structural properties of a pyroclastic soft rock. In: Proceedings of the Second International Symposium on hard soils-soft rocks 1998, Naples, pp. 85-91 (1998)

4. Cecconi, M., Viggiani, G., Rampello, S.: An experimental investigation of the mechanical behaviour of a pyroclastic soft rock. In: Proceedings of the Second International Symposium on Hard Soils-Soft Rocks 1998, Naples, pp. 473-482 (1998)

5. Cheng, Y.P., Nakata, Y., Bolton, M.D.: Discrete element simulation of crushable soil. Géotechnique 53(7), 633-641 (2003)

6. Cho, G.C., Dodds, L., Santamarina, J.C.: Particle shape effects on packing density, stiffness, and strength: natural and crushed sands. J. Geotech. Eng. ASCE 132(5), 591-602 (2006)

7. Coop, M.R., Sorensen, K.K., Bodas, Freitas T., Georgoutos, G.: Particle breakage during shearing of a carbonate sand. Géotechnique 54(3), 157-163 (2004)

8. Einav, I.: Breakage mechanics. Part 1: theory. J. Mech. Phys. Solids 55(6), 1274-1297 (2007)

9. Ezaoui, A., Lecompte, T., Di Benedetto, H., Garcia, E.: Effects of various loading stress paths on the stress-strain properties and on crushability of an industrial soft granular material. Granul. Matter 13, 283-301 (2011)

10. Fragaszy, R.J., Voss, M.E.: Undrained compression behavior of sand. J. Geotech. Eng. ASCE 112(3), 334-347 (1986)

11. Gagliardi, C.: Comportamento meccanico di un materiale granulare a grani frantumabili. Università di Roma Tor Vergata, Master Thesis (2003)

12. Han, G., Dusseault, M.B.: Description of fluid flow around a wellbore with stress-dependent porosity and permeability. J. Petrol. Sci. Eng. 40(1-2), 1-16 (2003)

13. Hardin, B.O.: Crushing of soil particles. J. Geotech. Eng. ASCE 111(10), 1177-1192 (1985)

14. Holm, T.A., Valsangkar, A.J.: Lightweight aggregate soil mechanics: properties and applications. ESCSI No. 6610 (2001)

15. Imre, B.: Micro mechanical Analyses of Sturzstroms (Rock Avalanches) on Earth and Mars. Dissertation No. 18854, ETH Zurich (2010)

16. Imre, B., Laue, J., Springman, S.M.: Fractal fragmentation of rocks within sturzstroms: insight derived from physical experiments within the ETH geotechnical drum centrifuge. Granul. Matter 12(3), 267-285 (2010)

17. Lackenby, J., Indraratna, B., McDowell, G., Christie, D.: Effect of confining pressure on ballast degradation and deformation under cyclic triaxial loading. Géotechnique 57(6), 527-536 (2007)
18. Lade, P.V., Yamamuro, J.A., Bopp, P.A.: Significance of particle crushing in granular materials. J. Geotech. Eng. ASCE 122(4), 309-316 (1996)

19. Lee, K.L. \& Farhoomand, I.: Compressibility and crushing of granular soils in anisotropic triaxial compression. Can. Geotech. J. 4(1), 68-86 (1967)

20. Lees, G.: A new method for determining the angularity of particles. Sedimentology 3(1), 2-21 (1964)

21. Leu, J., Low, B., Zimmermann, A.: Effects of grain size distribution and confining stress on the mechanical behaviour of an artificial crushable material. Semester thesis, ETH Zurich (2011)

22. Lobo, Guerrero S., Vallejo, L.E.: DEM analysis of crushing around driven piles in granular materials. Géotechnique 55(8), 617-623 (2005)

23. Luzzani, L., Coop, M.R.: On the relationship between particle breakage and the critical state of sands. Soils Found. 42(2), 7182 (2002)

24. McDowell, G.R.: A physical justification for $\log (\mathrm{e})-\log (\sigma)$ based on fractal crushing and particle kinematics. Géotechnique 55(9), 697-698 (2005)

25. McDowell, G.R., Bolton, M.D.: On the micromechanics of crushable aggregates. Géotechnique 40(5), 667-679 (1998)

26. McDowell, G.R., Bolton, M.D., Robertson, D.: The fractal crushing of granular materials. J. Mech. Phys. Solids 44(12), 2079-2102 (1996)

27. Miura, K., Maeda, K., Furukawa, M., Toki, S.: Physical characteristics of sands with different primary properties. Soils Found. 37(3), 53-64 (1997)

28. Nakata, Y., Hyde, A.F.L., Hyodo, M., Murata, H.: A probabilistic approach to sand particle crushing in the triaxial test. Géotechnique 49(5), 567-583 (1999)

29. Okada, Y., Sassa, K., Fukuoka, H.: Excess pore pressure and grain crushing of sands by means of undrained and naturally drained ring-shear tests. Eng. Geol. 75, 325-343 (2004)

30. Palmer, A.C., Sanderson, T.J.O.: Fractal crushing of ice and brittle solids. Proc. R. Soc. Lond. 433-460 (1991).

31. Papamichos, E., Vardoulakis, I., Ouadfel, H.: Permeability reduction due to grain crushing around a perforation. Int. J. Rock Mech. Mineral Sci. 30(7), 1223-1229 (1993)

32. Peck, A.: Beginning GIMP: From Novice to Professional, 2nd Edn, p. 548. Apress (2008)

33. Russell, A.R., Muir, Wood D.: Crushing of particles in idealised granular assemblies. J. Mech. Phys. Solids 57, 1293-1313 (2009)

34. Sammis, C.G., King, G., Biegel, R.: The kinematics of gouge deformations. Pure Appl. Geophys. 125, 777-812 (1987)

35. Simonini, P.: Analysis of behaviour of sand surrounding pile tips. J. Geotech. Eng. 122, 897-905 (1996)

36. Tarantino, A., Hyde, A.F.L.: An experimental investigation of work dissipation in crushable materials. Géotechnique 55(8), 575-584 (2005)

37. Turcotte, D.L.: Fractals and fragmentation. J. Geophys. Res. 91(B2), 1921-1926 (1986)

38. Vallejo, L.E., Lobo-Guerrero, S., Hammer, K.: Degradation of a granular base under a flexible pavement: DEM simulation. Int. J. Geomech. 6(6), 435-439 (2006)

39. Vallejo, L.E., Lobo-Guerrero, S.: The shear strength of granular materials containing dispersed oversized particles: DEM analysis. Int. J. Geotech. Eng. 6(3), 371-379 (2012)

40. Vallejo, L.E., Lobo-Guerrero, S., Chik, Z.: A network of fractal force chains and their effect in granular materials undercompression. In: Levy-Vehel, J., Lutton, E. (eds.) Fractals in Engineering, pp. 67-80. Springer, Berlin (2005)

41. Wanninger, F., Zwicker, P.: Effects of Grain Crushing on Compressibility of an Artificial Material. Semester Thesis, ETH Zurich (2010) 
42. White, D.J., Bolton, M.D.: Soil deformation around a displacement pile in sand. In: Proceedings of the International Conference on Physical Modelling in Geotechnics, St John's, Newfoundland. Balkema, Rotterdam, pp. 649-654 (2002)

43. Wood, D.M., Maeda, K.: Changing grading of soil: effect on critical states. Acta Geotech. 3, 3-14 (2008)
44. Yasufuku, N., Hyde, A.F.: Pile end bearing capacity in crushable sand. Géotechnique 45(4), 663-676 (1995)

45. Zheghal, M.: The impact of grain crushing on road performance. Geotech. Geol. Eng. 27, 549-558 (2009) 